\title{
AFAP1-AS1 induces cisplatin resistance in non-small cell lung cancer through PI3K/AKT pathway
}

\author{
YANG LIU ${ }^{1}$, QIANG $\mathrm{HU}^{2}$ and XIHUI WANG ${ }^{3}$ \\ Departments of ${ }^{1}$ Pharmacy, ${ }^{2}$ Radiology, and ${ }^{3}$ Blood Transfusion, Linyi Central Hospital, \\ Linyi, Shandong 276400, P.R. China
}

Received September 13, 2019; Accepted November 12, 2019

DOI: 10.3892/ol.2019.11175

\begin{abstract}
Cisplatin (DDP)-resistance in non-small cell lung carcinoma (NSCLC) severely influences the prognosis of affected patients. This study aims to uncover the potential role of AFAP1-AS1 in DDP-resistant NSCLC and the underlying mechanism. The expression level of AFAP1-AS1 in DDP-resistant NSCLC patients and DDP-resistant A549 cells (A549/DDP) was determined. Proliferative, cell cycle distribution, apoptotic, migratory and invasive changes in A549/DDP cells transfected with si-AFAP1-AS1 were assessed. Western blot analyses were conducted to examine the protein levels of phosphorylated protein kinase B (p-AKT), AKT, E-cadherin, N-cadherin, vimentin and snail in A549/DDP cells. Furthermore, the ubcellular distribution of AFAP1-AS1 was analyzed. Through RNA immunoprecipitation (RIP) assay, the interaction between AFAP1-AS1 and enhancer of zeste homolog 2 (EZH2) was explored. Finally, the regulatory effect of EZH2 on the PI3K/AKT pathway was investigated by western blot analysis. AFAP1-AS1 was upregulated in DDP-resistant NSCLC patients and A549/DDP cells. Transfection with si-AFAP1-AS1 attenuated the proliferative, migratory and invasive abilities, arrested cell cycle in G0/G1 phase, and stimulated apoptosis of A549/DDP cells. Silencing of AFAP1-AS1 upregulated E-cadherin and downregulated $\mathrm{N}$-cadherin, vimentin and snail expression levels. Furthermore, AFAP1-AS1 was verified to interact with EZH2. The relative expression of EZH2 was reduced by transfection of A549/DDP cells with si-AFAP1-AS1. Silencing of EZH2 inhibited the activation of PI3K/AKT pathway. In conclusion, AFAP1-AS1 accelerates the proliferative and metastatic abilities of A549/DDP cells, whereas inhibits the apoptosis of A549/DDP cells, by interacting with EZH2 to activate the $\mathrm{PI} 3 \mathrm{~K} / \mathrm{AKT}$ pathway; thus, inducing DDP resistance in NSCLC.
\end{abstract}

Correspondence to: Dr Xihui Wang, Department of Blood Transfusion, Linyi Central Hospital, 17 Jiankang Road, Yishui, Linyi, Shandong 276400, P.R. China

E-mail: xkhwq622@163.com

Key words: AFAP1-AS1, PI3K/AKT, non-small cell lung carcinoma, cisplatin resistance

\section{Introduction}

Lung carcinoma is the first leading malignancy in males, and its incidence in females is on the rise. Globally, lung carcinoma is a fetal malignant cancer that seriously endangers human lives (1). In China, the incidence of lung carcinoma ranks first in urban population and second in rural ones (2). Non-small cell lung carcinoma (NSCLC) is the majority subtype of lung carcinoma, accounting for $75-80 \%$ of all lung carcinoma cases. The prognosis of NSCLC is unsatisfactory despite the advanced progression in therapeutic strategies. Invasiveness and metastasis are the major reasons for the poor prognosis of NSCLC. Chemotherapy based on platinum-containing drugs is preferred for advanced NSCLC patients (3). Cisplatin (DDP) is the most common first-line platinum-based chemotherapy drug. DDP could inhibit DNA replication, affect cell transcription and translation, and promote apoptosis of tumor cells. However, some tumor patients experience DDP resistance during their chemotherapy treatment. Treatment failure of DDP-based chemotherapy remarkably affects the survival of tumor patients $(4,5)$. Thus, uncovering the mechanism underlying DDP resistance in tumors would improve the therapeutic efficacy of NSCLC.

As an important member of the non-coding RNA family, long non-coding RNA (IncRNA) regulates gene expression at epigenetic, transcriptional, and post-transcriptional levels, thereby affecting the progression of NCSLC (6). With the advances in high-throughput sequencing technology and bioinformatics analysis methods, a great number of lncRNAs with potential biological values in tumors have been identified. lncRNAs are capable of alleviating or inducing drug-resistance in tumors through a number of different mechanisms (7-9).

AFAP1-AS1 is a newly discovered lncRNA that encodes an antisense product of the AFAP1 gene. Studies have shown that AFAP1-AS1 exerts a carcinogenic role in numerous types of tumors. In lung carcinoma, AFAP1-AS1 is highly expressed and accelerates invasiveness and metastasis by inhibiting AFAP1 level (10). Upregulated level of AFAP1-AS1 in liver cancer is correlated to poor prognosis of affected patients through activating RhoA/Rac2 pathway (11). AFAP1-AS1 is upregulated in gastric cancer, which stimulates proliferation and suppresses apoptosis through PTEN/p-AKT pathway (12). Additionally, AFAP1-AS1 is significantly upregulated in esophageal adenocarcinoma tissues and cells. Knockdown 
of AFAP1-AS1 in esophageal adenocarcinoma attenuates the proliferative, migratory and invasive abilities, whereas induces apoptosis of tumor cells (13). However, the biological function of AFAP1-AS1 in DDP-resistant NSCLC has not been reported.

\section{Patients and methods}

Sample collection. Thirty-four tumor tissues of NSCLC patients were surgically resected from January 2011 to December 2017 in Linyi Central Hospital (Linyi, China). The patients included in the study were 23 males and 11 females between 54 and 78 years of age. According to the DDP resistance, the NSCLC tissues were divided into the DDP and NG group. Samples were immediately preserved in liquid nitrogen within 15 min ex vivo. The study was approved by the Ethics Committee of Linyi Central Hospital. Signed written informed consents were obtained from the patients and/or guardians.

Reverse transcription-quantitative polymerase chain reaction $(R T-q P C R)$. Total RNA was extracted from NSCLC tissues using TRIzol ${ }^{\circledR}$ reagent (Invitrogen; Thermo Fisher Scientific, Inc.), and was quantified using NanoDrop 2000 spectrophotometer (NanoDrop Technologies; Thermo Fisher Scientific, Inc.). Total RNA was reverse transcribed into complementary deoxyribonucleic acid (cDNA) at $37^{\circ} \mathrm{C}$ for $15 \mathrm{~min}$ using PrimeScript RT reagent (Takara Biotechnology Co., Ltd.). The obtained cDNA was further amplified by quantitative PCR using SYBR ${ }^{\circledR}$ Premix Ex Taq ${ }^{\mathrm{TM}}$ (Takara Biotechnology Co., Ltd.). The reaction system volume was $25 \mu 1$ in total. The following thermocycling conditions were used for PCR: pre-denaturation at $95^{\circ} \mathrm{C}$ for $5 \mathrm{~min}$, denaturation at $95^{\circ} \mathrm{C}$ for $30 \mathrm{sec}$, annealing at $60^{\circ} \mathrm{C}$ for $45 \mathrm{sec}$, extension at $72^{\circ} \mathrm{C}$ for $3 \mathrm{~min}$, for 35 cycles, and then extension at $72^{\circ} \mathrm{C}$ for $5 \mathrm{~min}$. PCR products were stored at $4^{\circ} \mathrm{C}$. The relative levels were quantitatively analyzed using the $2^{-\Delta \Delta \mathrm{Cq}}$ method (14). Glyceraldehyde 3-phosphate dehydrogenase (GAPDH) was used as internal reference. Primer sequences were as follows: AFAP1-AS1 forward, TCGCTCAATGGAGTGACGGCA and reverse, CGGCTGAGACCGCTGAGAACT; GAPDH forward, GGA GCGAGATCCCTCCAAAAT and reverse, GGCTGTTGT CATACTTCTCATGG; U6 forward, AAAATATGGAACGC TTCACGAATTTG and reverse, CTCGCTTCGGCAGCA CATATACT.

Cell culture. A549 cells (cat. no. SCSP-503) and DDP-resistant cell line A549/DDP (cat. no. SCSP-524) were provided by the Shanghai Cell Bank (http://www.cellbank.org.cn/). A549 cells were cultured in RPMI-1640 medium (HyClone; GE Healthcare Life Sciences) containing $10 \%$ fetal bovine serum (FBS) (Gibco; Thermo Fisher Scientific, Inc.). A549/DDP cells were cultured in medium containing $1 \mu \mathrm{mol} / 1$ DDP to maintain drug resistance.

Cell transfection. Cells in the logarithmic growth phase were subjected to transfection at confluency of $70-80 \%$. Transfection vectors and Lipofectamine ${ }^{\circledR} 2000$ (Invitrogen; Thermo Fisher Scientific, Inc.) were respectively diluted in Opti-MEM (Shanghai GeneChem Co., Ltd.). After mixture and incubation for $20 \mathrm{~min}$, the cells were plated in each well.
si-AFAP1-AS1 at the concentration of $50 \mathrm{nM}$ was added to each well and then incubated for $48 \mathrm{~h}$. Complete fresh medium was replaced after $6 \mathrm{~h}$. The transfection plasmids were provided by Shanghai GenePharma Co., Ltd.

Cell Counting Kit-8 (CCK-8). Cells were seeded in a 96-well plate with $2.0 \times 10^{3}$ cells/well and incubated with $0,0.5,1,1.5$, 2 and $2.5 \mathrm{mg} / \mathrm{ml}$ DDP, respectively. Each experiment was performed in triplicate. Absorbance at $450 \mathrm{~nm}$ was recorded using CCK-8 kit (Dojindo Molecular Technologies, Inc.), the viability curve was plotted and $\mathrm{IC}_{50}$ was calculated.

Colony formation assay. Cells were seeded in a 6-well plate with 500 cells/well and incubated for 2-3 weeks. Subsequently, the cells were fixed in $100 \%$ methanol at $20^{\circ} \mathrm{C}$ and dyed with $0.5 \%$ crystal violet (Sigma-Aldrich; Merck KGaA) at $20^{\circ} \mathrm{C}$ for $20 \mathrm{~min}$. Colonies of $>50 \mu \mathrm{m}$ in size were counted by Quantity One software (Bio-Rad Laboratories, Inc.). Colonies were finally observed under a light microscope (BX-42; Olympus Corporation) and calculated (magnification, $\mathrm{x} 10$ ).

Cell cycle determination. Transfected cells for $48 \mathrm{~h}$ were washed with pre-cold PBS and fixed in pre-cold ethanol overnight. Subsequently, the cells were subjected to incubation at $20^{\circ} \mathrm{C}$ for $10 \mathrm{~min}$ with $10 \mu \mathrm{l}$ of propidium iodide (PI) and RNase $\mathrm{A}$ in the dark for $1 \mathrm{~h}$. Cell cycle was determined using a flow cytometer (FACSCalibur; BD Biosciences). Data were obtained and analyzed using the CellQuest Pro software (version 3.3; Becton, Dickinson and Company).

Cell apoptosis determination. Cells were resuspended in $500 \mu \mathrm{l}$ of binding buffer at the dose of $1-5 \times 10^{5} / \mathrm{ml}$. Subsequently, the cells were subjected to incubation with $5 \mu \mathrm{l}$ of Annexin V-FITC and $5 \mu \mathrm{l}$ of PI in the dark at $20^{\circ} \mathrm{C}$ for $30 \mathrm{~min}$. Apoptosis was determined using flow cytometry within half an hour. Flow cytometer (FACSCalibur) was used for analysis. Data were obtained and analyzed using the CellQuest Pro software (version 3.3).

Western blot analysis. The cells were lysed using a cell lysis buffer (cat. no. QC25-05099; Shanghai Qincheng Biotechnology, Co.). Total protein was extracted from cells using radioimmunoprecipitation assay and was quantified using the bicinchoninic acid (both from Beyotime Institute of Biotechnology) method. A total of $30 \mu \mathrm{g}$ of protein was added per lane for electrophoresis. The extracted protein was separated using a 10\% SDS-PAGE gel. After transferred onto polyvinylidene fluoride membranes (EMD Millipore), the protein was blocked in $5 \%$ skim milk at $4^{\circ} \mathrm{C}$ for $2 \mathrm{~h}$, incubated with primary antibodies at $4^{\circ} \mathrm{C}$ overnight and secondary antibodies at $20^{\circ} \mathrm{C}$ for $2 \mathrm{~h}$. Bands were visualized by an enhanced chemiluminescence detection kit (Amersham; GE Healthcare) and analyzed by Image J Software (National Institutes of Health). Rabbit polyclonal Akt antibody (dilution, 1:500; cat. no. ab8805), rabbit monoclonal p-Akt antibody (dilution, 1:500; cat. no. ab81283), rabbit polyclonal E-cadherin antibody (dilution, 1:500; cat. no. ab15148), rabbit polyclonal N-cadherin antibody (dilution, 1:500; cat. no. ab18203), rabbit monoclonal snail antibody (dilution, 1:500; cat. no. ab216347), rabbit polyclonal vimentin antibody (dilution, 1:500; cat. no. ab137321), 
A

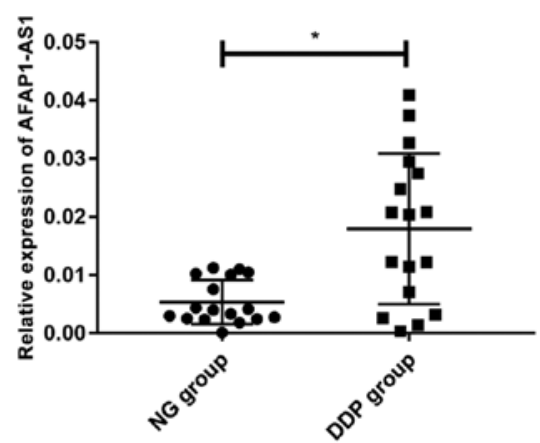

C

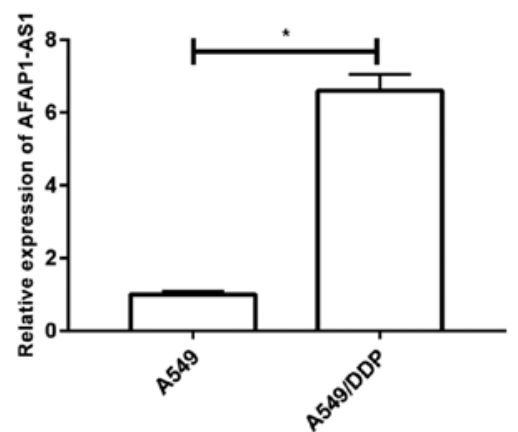

B

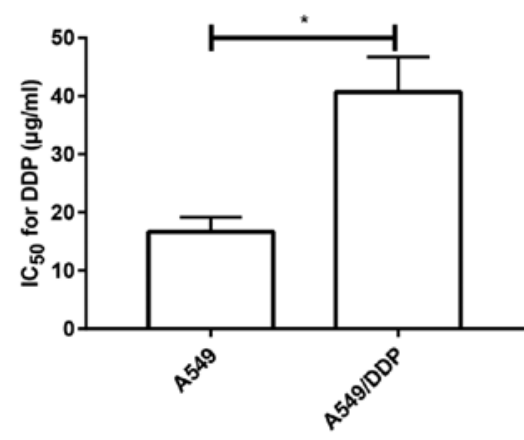

D

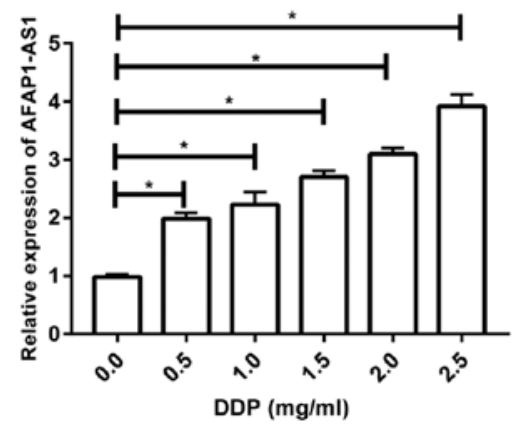

Figure 1. AFAP1-AS1 was upregulated in DDP-resistant NSCLC patients and cell lines. (A) Relative expression of AFAP1-AS1 in NSCLC patients who did not receive DDP-based chemotherapy and those with DDP-resistance. (B) IC 50 for DDP in A549 and A549/DDP cells. (C) Relative expression of AFAP1-AS1 in A549 and A549/DDP cells. (D) AFAP1-AS1 expression in A549 cells treated with medium containing 0, 0.5, 1, 1.5, 2 and 2.5 mg/ml DDP for 24 h. * $<0.05$. DDP, cisplatin; NSCLC, non-small cell lung carcinoma.

rabbit polyclonal GAPDH antibody (dilution, 1:500; cat. no. ab37168) and secondary goat anti-rabbit (HRP) IgG antibody (dilution, 1:2,000; cat. no. ab6721) were all purchased from Abcam. GAPDH was used as the reference protein.

Transwell assay. Cell density was adjusted to $1-5 \times 10^{5} / \mathrm{ml}$. Suspension (200 $\mu \mathrm{l} /$ well) was applied in the upper chamber of the Matrigel-coated (diluted in serum-free medium with 1:10) Transwell chamber (EMD Millipore). In the bottom chamber, $500 \mu \mathrm{l}$ of medium containing $10 \%$ FBS were applied. The cells on the top surface of the membrane were removed by a cotton swab after a $24-\mathrm{h}$ incubation at $37^{\circ} \mathrm{C}$. After incubation for $48 \mathrm{~h}$, the invasive cells were fixed in $4 \%$ paraformaldehyde, dyed with crystal violet for $2 \mathrm{~min}$ at $37^{\circ} \mathrm{C}$ and counted using a light microscope (BX-42; Olympus Corporation). Penetrating cells were counted in ten randomly selected fields per sample (magnification, $\mathrm{x} 10$ ). Transwell migration assay was conducted following the same procedures except for Matrigel pre-coating.

Subcellular distribution analysis. Cytoplasmic and nuclear RNA was extracted using the PARIS kit (Invitrogen; Thermo Fisher Scientific, Inc.) and then subjected to RT-qPCR. U6 and GAPDH were the internal references of nucleus and cytoplasm, respectively.

RNA immunoprecipitation $(R I P)$. Cells were treated following the manufacturer's instructions of Millipore Magna RIP ${ }^{\mathrm{TM}}$ RNA-Binding Protein Immunoprecipitation kit (EMD
Millipore). Cell lysate was incubated with rabbit monoclonal enhancer of zeste homolog 2 (EZH2) antibody (dilution, 1:500; cat. no. ab191080; Abcam) or anti-IgG antibody at $4^{\circ} \mathrm{C}$ for $6 \mathrm{~h}$. A protein-RNA complex was captured and digested with $0.5 \mathrm{mg} / \mathrm{ml}$ proteinase $\mathrm{K}$ containing $0.1 \%$ SDS to extract RNA. The magnetic beads were repeatedly washed with RIP washing buffer to remove non-specific adsorption as much as possible. Finally, the extracted RNA was subjected to mRNA level determination using RT-qPCR.

Statistical analysis. SPSS 19.0 software (IBM Corp.) was used for data analysis. Data were expressed as the mean \pm standard deviation. Intergroup comparisons were made using t-test. Comparisons between multiple groups were made using one-way ANOVA followed by the Least Significant Difference post hoc test. $\mathrm{P}<0.05$ was considered to indicate a statistically significant difference.

\section{Results}

AFAP1-AS1 is upregulated in DDP-resistant NSCLC patients and cell lines. Compared with the NSCLC patients who did not receive DDP-based chemotherapy, those with DDP resistance presented higher level of AFAP1-AS1 (Fig. 1A). CCK-8 assay revealed a higher $\mathrm{IC}_{50}$ for DDP in A549/DDP cells compared with that in A549 cells, verifying the cytotoxicity in DDP-resistant A549 cell line (Fig. 1B). Similarly, AFAP1-AS1 expression was much higher in A549/DDP cells than that in the 
A

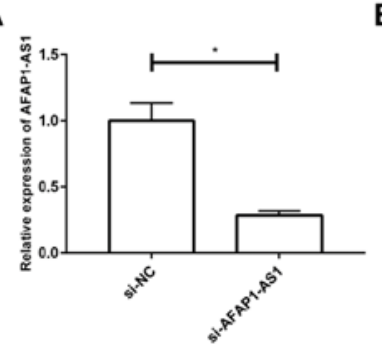

B
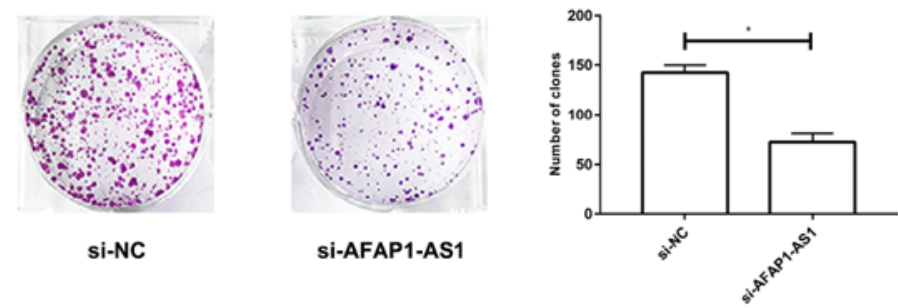

C

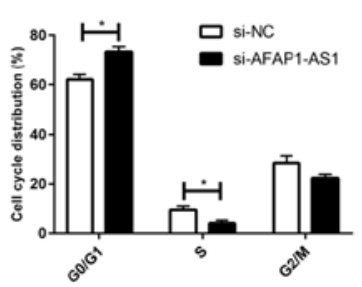

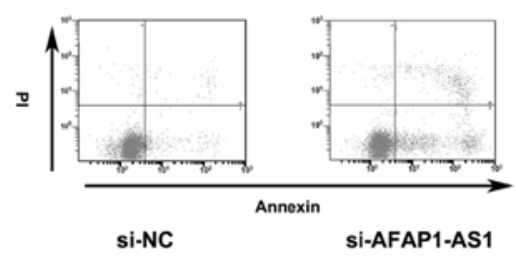

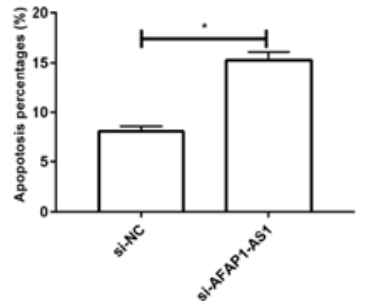

E
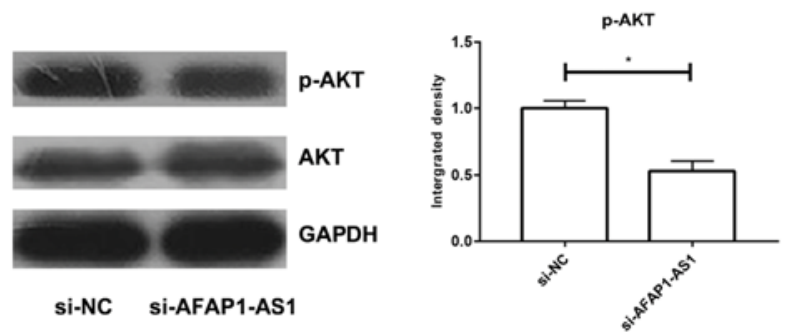

Figure 2. Silencing of AFAP1-AS1 suppressed the proliferative ability and cell cycle progression, and induced apoptosis in A549/DDP cells by PI3K/AKT pathway. (A) Transfection efficacy of si-AFAP1-AS1 in A549/DDP cells. (B) Colony formation assay revealed the number of colonies in A549/DDP cells transfected with si-NC or si-AFAP1-AS1. (C) Cell cycle distribution in A549/DDP cells transfected with si-NC or si-AFAP1-AS1. (D) Apoptotic rate in A549/DDP cells transfected with si-NC or si-AFAP1-AS1. (E) Western blot analysis was used for the protein expression levels of p-AKT and AKT in A549/DDP cells transfected with si-NC or si-AFAP1-AS1. ${ }^{\text {P }}<0.05$. DDP, cisplatin; GAPDH, glyceraldehyde 3-phosphate dehydrogenase.

parental cells (Fig. 1C). A549 cells were cultured in medium containing $0,0.5,1,1.5,2$ and $2.5 \mathrm{mg} / \mathrm{ml}$ DDP for $24 \mathrm{~h}$. RT-qPCR revealed that the AFAP1-AS1 expression gradually increased in a dose-dependent manner, indicating the involvement of AFAP1-AS1 in DDP-resistant NSCLC (Fig. 1D).

Silencing of AFAP1-AS1 suppresses the proliferative ability and cell cycle progression, and induces apoptosis in A549/DDP cells through the PI3K/AKT pathway. To uncover the biological function of AFAP1-AS1 in DDP-resistant NSCLC, si-AFAP1-AS1 was constructed and its transfection efficacy in A549/DDP cells was tested (Fig. 2A). Transfection with si-AFAP1-AS1 markedly decreased the number of colonies in A549/DDP cells and arrested the cells in G0/G1 phase (Fig. 2B and C). Moreover, silencing of AFAP1-AS1 greatly enhanced the apoptotic rate in DDP-resistant cells (Fig. 2D). Western blot analysis revealed the downregulation of p-AKT in A549/DDP cells transfected with si-AFAP1-AS1, confirming the inhibited PI3K/AKT pathway (Fig. 2E).

Silencing of AFAP1-AS1 suppresses migratory and invasive abilities of A549/DDP cells by inhibiting epithelial-mesenchymal transition (EMT). Transfection with si-AFAP1-AS1 attenuated the migratory and invasive abilities of A549/DDP cells (Fig. 3A and B). Since EMT is vital in tumor metastasis, key proteins related to EMT were examined. Silencing of AFAP1-AS1 upregulated E-cadherin; however, downregulated N-cadherin, vimentin and snail (Fig. 3C). These results suggest that knockdown of AFAP1-AS1 markedly inhibits EMT in DDP-resistant NSCLC.

AFAP1-AS1 activates PI3K/AKT pathway through interacting with $E Z H 2$. Subcellular distribution analysis revealed that
AFAP1-AS1 was mainly expressed in nucleus (Fig. 4A). A previous study has presented that $\mathrm{EZH} 2$ induces the activation of PI3K/AKT pathway in NSCLC (15). Herein, RIP assay revealed higher enrichment of AFAP1-AS1 in anti-EZH2 compared with that in anti-IgG, confirming the interaction between AFAP1-AS1 and EZH2 (Fig. 4B). Transfection with si-AFAP1-AS1 sufficiently downregulated the protein level of EZH2, presenting a negative association (Fig. 4C). After transfection with si-EZH2, the protein level of p-AKT was remarkably downregulated, confirming the inhibited PI3K/AKT pathway (Fig. 4D).

\section{Discussion}

In the present study, abnormally expressed AFAP1-AS1 was found to be related to DDP resistance in NSCLC. AFAP1-AS1 level remained higher in DDP-resistant NSCLC tissues and cell lines. Silencing of AFAP1-AS1 suppressed proliferative, migratory and invasive abilities of A549/DDP cells through regulating EMT and PI3K/AKT pathway.

EMT is the process where glandular epithelial cells transform to mesenchymal cells. EMT enhances the migratory and invasive abilities, and attenuates aging and apoptosis of cells, thus improving the immunosuppression. EMT is a vital mechanism leading to distant metastasis of tumors. Once EMT occurs, downregulated E-cadherin disrupts the cell conjunction between adjacent epithelial cells, dislocates in situ tumor cells and thereafter results in tumor metastasis $(16,17)$. A variety of lncRNAs have been found to induce EMT in human tumors. lncRNA XIST induces EMT in osteosarcoma through targeting miR-195-5p/YAP axis (18). IncRNA XIST also stimulates the progression of EMT in NSCLC via regulating miR-367/miR-141-ZEB2 axis (19). IncRNA DNM3OS has been reported to participate in the EMT of ovarian cancer (20). Upregulation of DNM3OS stimulates the 
A

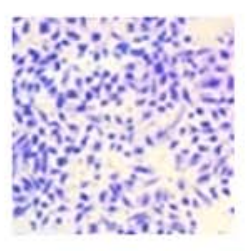

si-NC

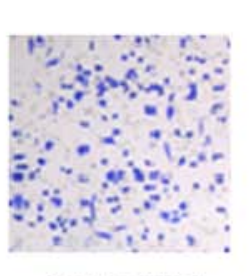

si-AFAP1-AS1

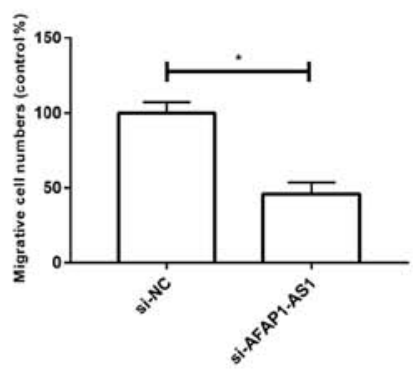

C

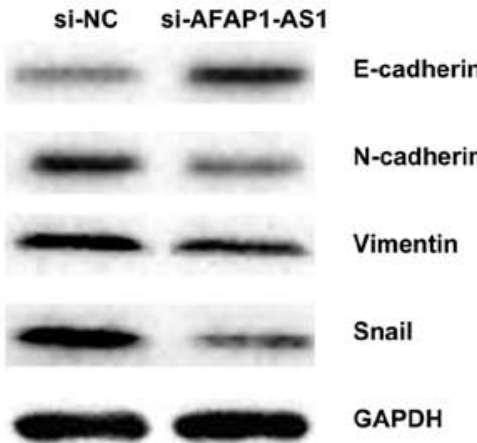

B

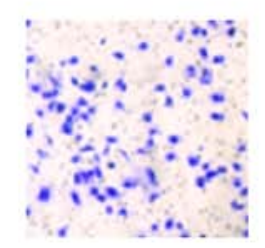

si-NC

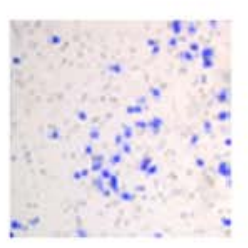

si-AFAP1-AS1
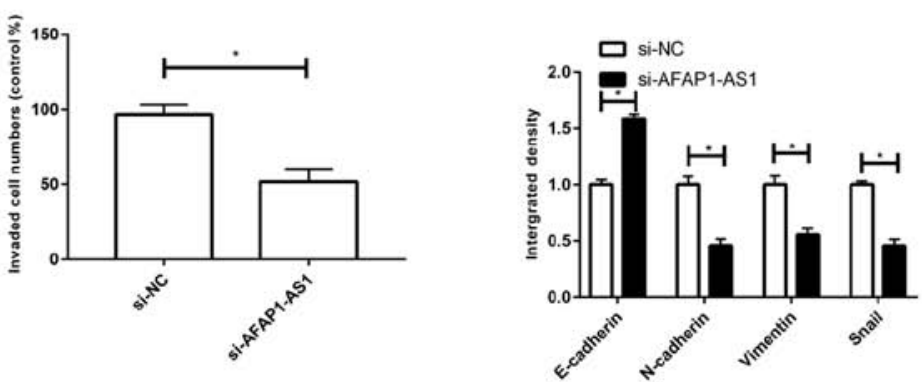

Figure 3. Silencing of AFAP1-AS1 suppressed the migratory and invasive abilities of A549/DDP cells by inhibiting EMT. Transwell assay revealed the (A) migration and (B) invasion in A549/DDP cells transfected with si-NC or si-AFAP1-AS1. (C) Western blot analysis was used for the protein expression levels of E-cadherin, $\mathrm{N}$-cadherin, vimentin and snail in A549/DDP cells transfected with si-NC or si-AFAP1-AS1. "P<0.05. DDP, cisplatin; EMT, epithelial-mesenchymal transition; GAPDH, glyceraldehyde 3-phosphate dehydrogenase.

A

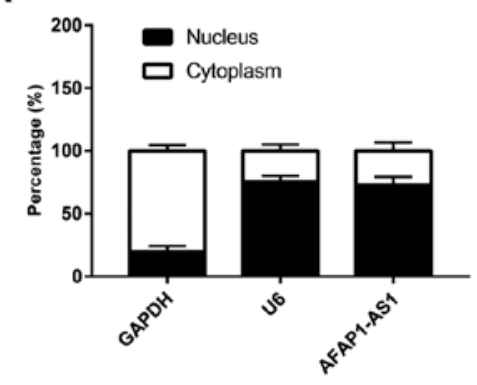

B

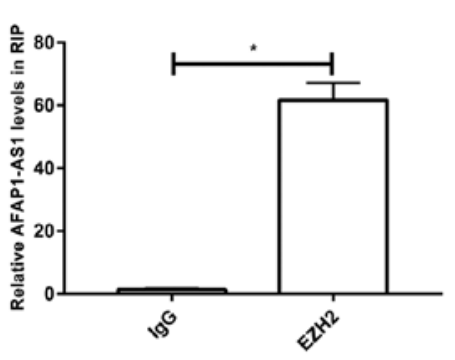

C
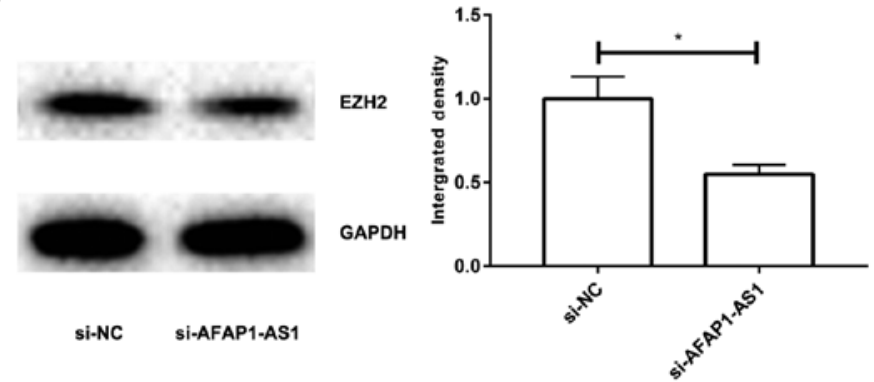

D

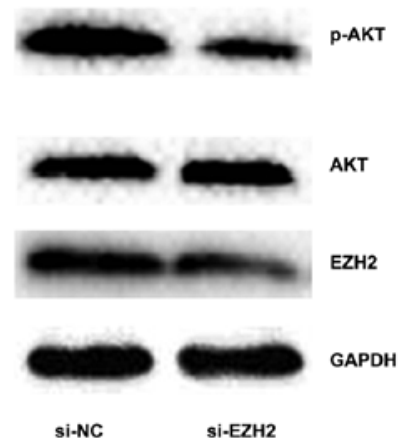

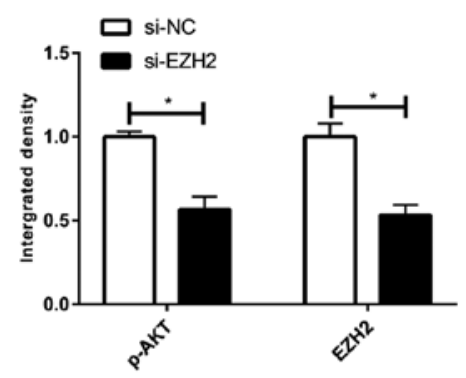

Figure 4. AFAP1-AS1 activated PI3K/AKT pathway through interacting with EZH2. (A) Subcellular distribution of AFAP1-AS1 in cytoplasm and nucleus of A549/DDP cells. GAPDH and U6 were internal references for cytoplasm and nucleus, respectively. (B) RIP assay showed the enrichment of AFAP1-AS1 in anti-IgG and anti-EZH2. (C) Western blot analysis of EZH2 expression in A549/DDP cells transfected with si-NC or si-AFAP1-AS1. (D) Western blot analysis of p-AKT, AKT and EZH2 in A549/DDP cells transfected with si-NC or si-EZH2. "P<0.05. EZH2, enhancer of zeste homolog 2; GAPDH, glyceraldehyde 3-phosphate dehydrogenase; RIP, RNA immunoprecipitation.

metastatic capacity of ovarian cancer cells and predicts a poor prognosis (20). In colorectal cancer, IncRNA N-BLR promotes
EMT and metastasis of tumor cells by mediating EMT-related transcriptional factor Zeb1 (21). In the present study, silencing of 
AFAP1-AS1 upregulated E-cadherin, whereas downregulated $\mathrm{N}$-cadherin, vimentin and snail, suggesting that AFAP1-AS1 markedly induces EMT in DDP-resistant NSCLC.

Transfection of A549/DDP cells with si-AFAP1-AS1 downregulated p-AKT level, indicating the involvement of PI3K/AKT pathway in DDP-resistant NSCLC. PI3K/AKT pathway is involved in regulating multiple cellular behaviors and tumor progression. PI3K/AKT pathway could be influenced by multiple factors in tumor diseases. For example, miR-221 activates PI3K/AKT pathway by negatively regulating PTEN, thereby mediating gefitinib sensitivity in cervical cancer (22). miR-146b aggravates the progression of thyroid cancer via targeting PI3K/AKT pathway (23). Recent studies have reported that numerous IncRNAs could be able to mediate PI3K/AKT pathway and thereafter aggravate tumor progression as oncogenes. For example, lncRNA ASAP1-IT1 attenuates malignant phenotypes of NSCLC through targeting PTEN/AKT pathway (24). The growth of osteosarcoma is stimulated by lncRNA UCA1 through activating PTEN/AKT pathway (25). The proliferation and EMT of NSCLC cells are promoted through lncRNA FAL1-induced activation of PTEN/AKT pathway (26). The results of the present study demonstrated that AFAP1-AS1 robustly interacts with EZH2 to activate PI3K/AKT pathway.

This study only constructed the knockdown model of AFAP1-AS1 in A549/DDP cells. In future research, an overexpression model of AFAP1-AS1 using lentiviruses or overexpression plasmids is required to confirm the conclusions of this study.

In conclusion, AFAP1-AS1 accelerates the proliferative and metastatic abilities of the A549/DDP cells, whereas inhibits the apoptosis of the A549/DDP cells by interacting with EZH2 to activate PI3K/AKT pathway; thus, inducing DDP resistance in NSCLC.

\section{Acknowledgements}

Not applicable.

\section{Funding}

No funding was received.

\section{Availability of data and materials}

All data generated or analyzed during this study are included in this published article.

\section{Authors' contributions}

YL and XW designed the study and performed the experiments, YL and QH acquired the data, XW and QH analyzed the data, YL and XW prepared the manuscript. All authors read and approved the final manuscript.

\section{Ethics approval and consent to participate}

The study was approved by the Ethics Committee of Linyi Central Hospital (Linyi, China). Signed written informed consents were obtained from the patients and/or guardians.

\section{Patient consent for publication}

Not applicable.

\section{Competing interests}

The authors declare that they have no competing interests.

\section{References}

1. Jemal A, Siegel R, Xu J and Ward E: Cancer statistics, 2010. CA Cancer J Clin 60: 277-300, 2010.

2. Zhao P, Dai M, Chen W and Li N: Cancer trends in China. Jpn J Clin Oncol 40: 281-285, 2010.

3. Zappa C and Mousa SA: Non-small cell lung cancer: Current treatment and future advances. Transl Lung Cancer Res 5: 288-300, 2016.

4. Xiong Y, Huang BY and Yin JY: Pharmacogenomics of platinum-based chemotherapy in non-small cell lung cancer: Focusing on DNA repair systems. Med Oncol 34: 48, 2017.

5. Tièche CC, Peng RW, Dorn P, Froment L, Schmid RA and Marti TM: Prolonged pemetrexed pretreatment augments persistence of cisplatin-induced DNA damage and eliminates resistant lung cancer stem-like cells associated with EMT. BMC Cancer 16: 125, 2016.

6. Zhan Y, Zang H,Feng J, Lu J, Chen L and Fan S: Long non-coding RNAs associated with non-small cell lung cancer. Oncotarget 8: 69174-69184, 2017.

7. Pan JJ, Xie XJ, Li X and Chen W: Long non-coding RNAs and drug resistance. Asian Pac J Cancer Prev 16: 8067-8073, 2015.

8. Wang H, Guan Z, He K, Qian J, Cao J and Teng L: LncRNA UCA1 in anti-cancer drug resistance. Oncotarget 8: 64638-64650, 2017.

9. Loewen G, Jayawickramarajah J, Zhuo Y and Shan B: Functions of lncRNA HOTAIR in lung cancer. J Hematol Oncol 7: 90, 2014.

10. Zeng Z, Bo H, Gong Z, Lian Y, Li X, Li X, Zhang W, Deng H, Zhou M, Peng S, et al: AFAP1-AS1, a long noncoding RNA upregulated in lung cancer and promotes invasion and metastasis. Tumour Biol 37: 729-737, 2016.

11. Zhang JY, Weng MZ, Song FB, Xu YG, Liu Q, Wu JY, Qin J, Jin T and Xu JM: Long noncoding RNA AFAP1-AS1 indicates a poor prognosis of hepatocellular carcinoma and promotes cell proliferation and invasion via upregulation of the RhoA/Rac2 signaling. Int J Oncol 48: 1590-1598, 2016.

12. Guo JQ, Li SJ and Guo GX: Long noncoding RNA AFAP1-AS1 promotes cell proliferation and apoptosis of gastric cancer cells via PTEN/p-AKT pathway. Dig Dis Sci 62: 2004-2010, 2017.

13. Wu W, Bhagat TD, Yang X, Song JH, Cheng Y, Agarwal R, Abraham JM, Ibrahim S, Bartenstein M, Hussain Z, et al: Hypomethylation of noncoding DNA regions and overexpression of the long noncoding RNA, AFAP1-AS1, in Barrett's esophagus and esophageal adenocarcinoma. Gastroenterology 144: 956-966.e4, 2013.

14. Livak KJ and Schmittgen TD: Analysis of relative gene expression data using real-time quantitative PCR and the 2(-Delta Delta C(T)) method. Methods 25: 402-408, 2001.

15. Geng J, Li X, Zhou Z, Wu CL, Dai M and Bai X: EZH2 promotes tumor progression via regulating VEGF-A/AKT signaling in non-small cell lung cancer. Cancer Lett 359: 275-287, 2015.

16. Brabletz T, Kalluri R, Nieto MA and Weinberg RA: EMT in cancer. Nat Rev Cancer 18: 128-134, 2018.

17. Roche J: The epithelial-to-mesenchymal transition in cancer. Cancers (Basel) 10: pii: E52, 2018.

18. Yang $\mathrm{C}$, Wu $\mathrm{K}$, Wang $\mathrm{S}$ and Wei G: Long non-coding RNA XIST promotes osteosarcoma progression by targeting YAP via miR-195-5p. J Cell Biochem 119: 5646-5656, 2018.

19. Li C, Wan L, Liu Z, Xu G, Wang S, Su Z, Zhang Y, Zhang C, Liu X, Lei Z, et al: Long non-coding RNA XIST promotes TGF- $\beta$-induced epithelial-mesenchymal transition by regulating miR-367/141-ZEB2 axis in non-small-cell lung cancer. Cancer Lett 418: 185-195, 2018.

20. Mitra R, Chen X, Greenawalt EJ, Maulik U, Jiang W, Zhao Z and Eischen CM: Decoding critical long non-coding RNA in ovarian cancer epithelial-to-mesenchymal transition. Nat Commun 8: 1604, 2017. 
21. Rigoutsos I, Lee SK, Nam SY, Anfossi S, Pasculli B, Pichler M, Jing Y, Rodriguez-Aguayo C, Telonis AG, Rossi S, et al: N-BLR, a primate-specific non-coding transcript leads to colorectal cancer invasion and migration. Genome Biol 18: 98, 2017.

22. Du J, Wang L, Li C, Yang H, Li Y, Hu H, Li H and Zhang Z MicroRNA-221 targets PTEN to reduce the sensitivity of cervical cancer cells to gefitinib through the PI3K/Akt signaling pathway. Tumour Biol 37: 3939-3947, 2016.

23. Ramírez-Moya J, Wert-Lamas L and Santisteban P: MicroRNA$146 \mathrm{~b}$ promotes PI3K/AKT pathway hyperactivation and thyroid cancer progression by targeting PTEN. Oncogene 37: 3369-3383, 2018.

24. Zhang L, Shi SB, Zhu Y, Qian TT and Wang HL: Long non-coding RNA ASAP1-IT1 promotes cell proliferation, invasion and metastasis through the PTEN/AKT signaling axis in non-small cell lung cancer. Eur Rev Med Pharmacol Sci 22: 142-149, 2018.
25. Li T, Xiao Y and Huang T: HIF-1 $\alpha$-induced upregulation of lncRNA UCA1 promotes cell growth in osteosarcoma by inactivating the PTEN/AKT signaling pathway. Oncol Rep 39: 1072-1080, 2018

26. Pan C, Yao G, Liu B, Ma T, Xia Y, Wei K, Wang J, Xu J, Chen L and Chen Y: Long noncoding RNA FAL1 promotes cell proliferation, invasion and epithelial-mesenchymal transition through the PTEN/AKT signaling axis in non-small cell lung cancer. Cell Physiol Biochem 43: 339-352, 2017.

(i)(9) This work is licensed under a Creative Commons EY NO ND Attribution-NonCommercial-NoDerivatives 4.0 International (CC BY-NC-ND 4.0) License. 